\title{
LabVIEW - Teaching tool for control design subject
}

\author{
Poonam Waje Mogal ${ }^{1}$, Mrs. Nilima Warke ${ }^{2}$ \\ ${ }^{I}$ (Instrumentation Department, R.A.I.T, Nerul, Navi Mumbai, Mumbai University, India) \\ 2 (Instrumentation Department, V.E.S.I.T., Chembur, Mumbai, Mumbai University, India)
}

\begin{abstract}
In this paper we take an opportunity to represent the use of laboratory practices for teachers and students to teach and learn a control design subject. We want to demonstrate use of LabVIEW control design toolkit for teachers and students. Students will find the control design subject easier and interesting by simulating the experimental results, analysing comparisons and the validation of the modelling and controller design. By just changing the parameters in the system equation students will come to know the comparison among different systems and they will visualise the different effects of different systems in one glance.

Using this method, the learning-teaching process will become an active one.
\end{abstract}

Keywords: active learning, Control Design Toolkit, graphical language, LabVIEW, virtual instrumentation.

\section{Introduction}

In order to make engineering, and more specifically control Engineering more attractive and engaging to students, new tools and methodologies are required. Active learning is one of those methodologies and Virtual Instrumentation (VI) is one of the key enabling technologies. Active learning refers to a methodology that allows students to learn by experimental validation (hands-on learning).

The inclusion of software simulation in control engineering education has many benefits to the students. Software simulations allow students to easily modify parameters and get instant results, thus allowing them to quickly connect theory to practice. Hands-on learning can greatly enhance the students' learning experience. First of all, engineering students tend to be visual, sensing, inductive, and active learners, so hands-on education lends naturally to the way engineering students learn.

National Instruments' LabVIEW (short for Laboratory Virtual Instrumentation Engineering Workbench) is based on the concept of data flow programming and is particularly suited to test and measurement applications. The three important components of such applications are data acquisition, data analysis and data visualization. LabVIEW offers an environment which covers these vital components [1].

\section{Objectives of LabVIEW as Learning and Teaching}

The main difficulty in teaching and learning of control design subject is that if the two systems have to be compared then their outputs should be taken differently and this is very time consuming in a lecture. It is not possible for every time to make out outputs of each and every system on a paper to students for analyzing the system performance of different systems. So there should be some technique through which it will become easier to see the performances of different systems and comparison of those by just changing the system equation. And this is done by LabVIEW software.

The benefits of simulation training and the use of LabVIEW control panels became evident in the laboratory experiments. As education and technology merge, the opportunities for teaching and learning expand even more. However, the very rapid rate of change in the fields of technology poses special problems for academic institutions, specifically for the engineering disciplines. There is a need of providing for students meaningful and relevant practical experiences while being limited by very finite resources in the provision of laboratory hardware and infrastructure. One solution to this problem is to use computer based techniques to interface the students with the physical world, with suitable front end design to provide increasing sophistication and increased flexibility. Recent developments and applications, specifically the LabVIEW-based applications, have shown that many pure lecture-based engineering courses and conventional experiments which are heavily dependent upon specialised instruments can be updated and integrated with custom-written virtual instrumentation (VI), and can be delivered by computers. It is a graphical programming language that allows engineers and scientists to develop their own virtual instrument, which is flexible, modular and economical [2].

The major change occurring at the present is the increasing number of user-friendly software that makes it possible for students to experience new and fast ways of learning. It is imperative that engineering courses give emphasis on design oriented laboratory hands-on experience in their programs. The LabVIEW is visual software developed in a graphical language for instrumentation and control. With LabVIEW, computer laboratory experiments such as measurements and control tasks are easily performed [3]. 


\section{The Control Design Toolkit}

The Control Design Toolkit was initially launched in Spring 2004. It expands LabVIEW's capabilities for control system and dynamic system analysis and design considerably. The set of functions available is comparable with the Control System Toolbox in Matlab and the similar control system function category in Octave.

The contents of the control design palette:

Once the Control Design Toolkit is installed, the Control Design palette is available from the Functions palette. The Control Design palette is shown in the figure below.

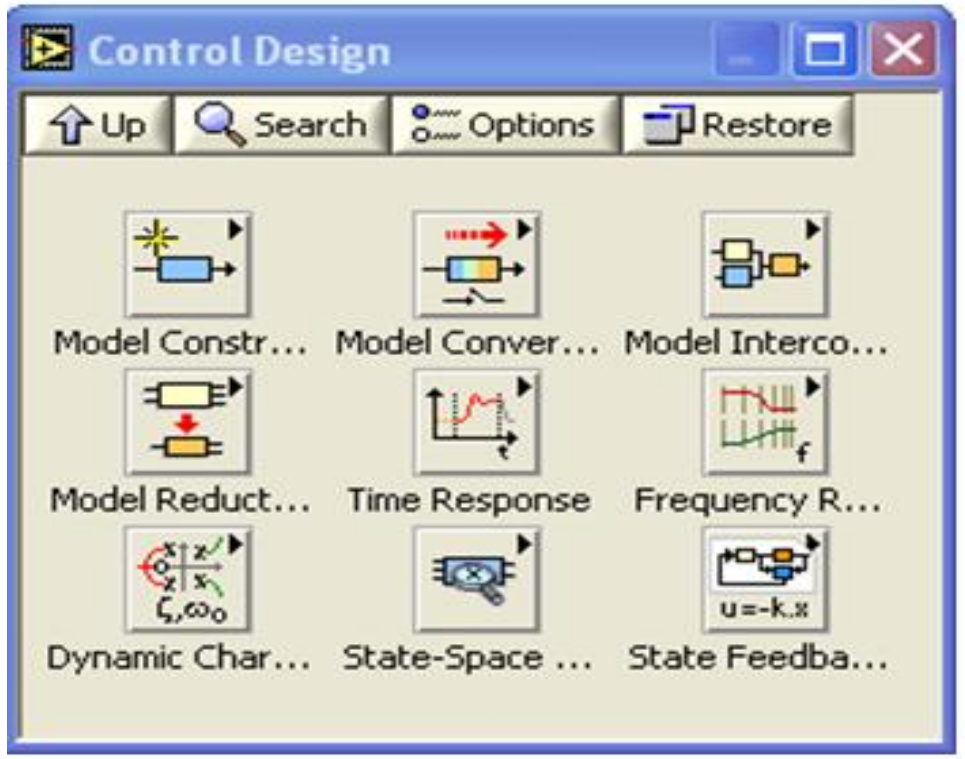

Fig.1 The Control Design palette

The Control Design and Simulation Module provides an interactive Control Design Assistant, a library of VIs, and a library of MathScript functions for designing a controller based on a model of a plant. The Control Design and Simulation Module also provides VIs that you can use to create and develop control design applications in LabVIEW. You can use these VIs to develop mathematical models of a dynamic system, analyze the models to learn about their dynamic characteristics, and create controllers to achieve specified dynamic characteristics [4].

\section{Features of control design toolkit}

- Design control systems using interactive graphical tools such as root-locus plots.

- Analyze system performance with tools such as pole-zero maps, step response graphs, and Bode plots.

- Integrate these control models with the NI LabVIEW Simulation Module.

- Construct plant and control models in state-space, transfer function, or pole-zero-gain form.

- Transform state -space model into transfer function and vice versa.

- Integrate with other LabVIEW modules such as LabVIEW State chart and LabVIEW Real-Time.

- Deploy dynamic systems to real-time hardware using built-in functions and LabVIEW Real-Time Module.

- Support for Windows 7/Vista/XP [5].

\section{Examples Using Control Design Toolkit}

\section{Calculating transfer function from state-space model}

The VI shown below shows how to get the SISO transfer function from input u to output y from the state-space model

where the system matrices are as shown in Fig.2.

$$
\begin{gathered}
d x / d t=A x+B u \\
y=C x+D u
\end{gathered}
$$



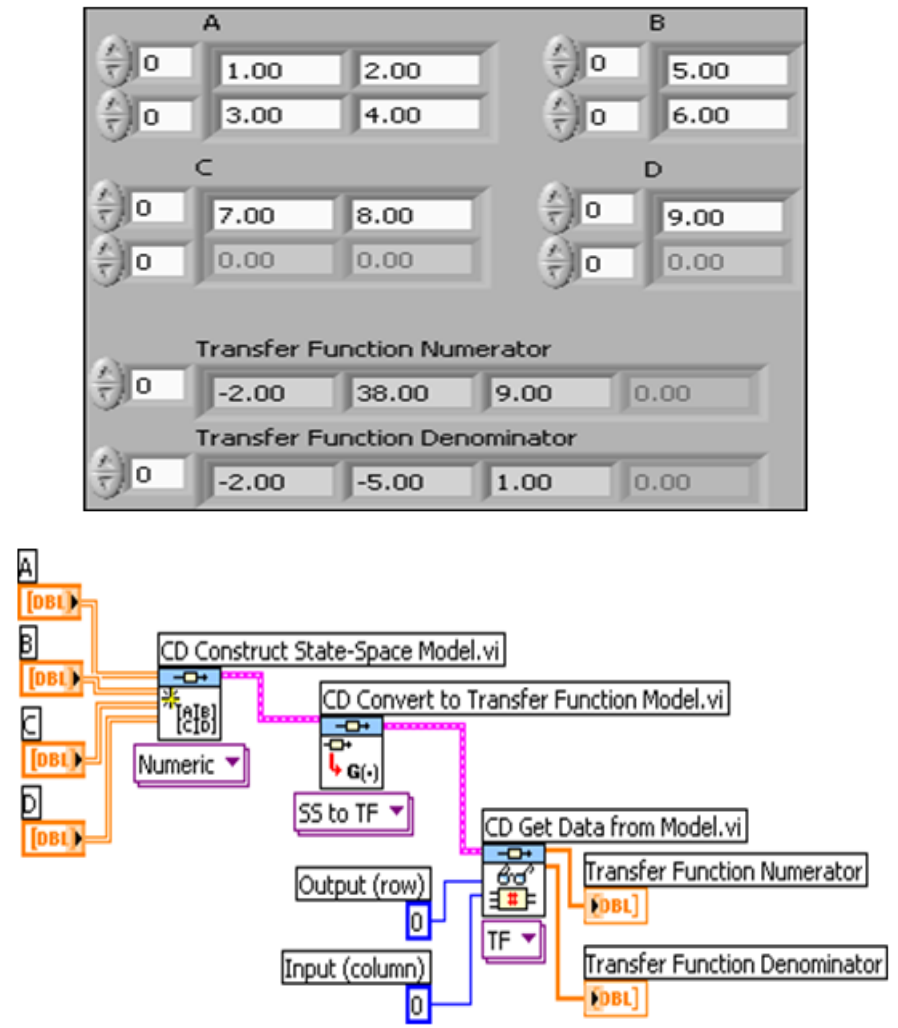

Frequency Response

Fig.2 Front panel and Block diagram of convert_ss_to_tf.vi.

The Frequency Response palette contains several functions for generating and plotting frequency response data for continuous-time as well as discrete-time models.

Frequency response of a continuous-time transfer function is shown in Fig.3.

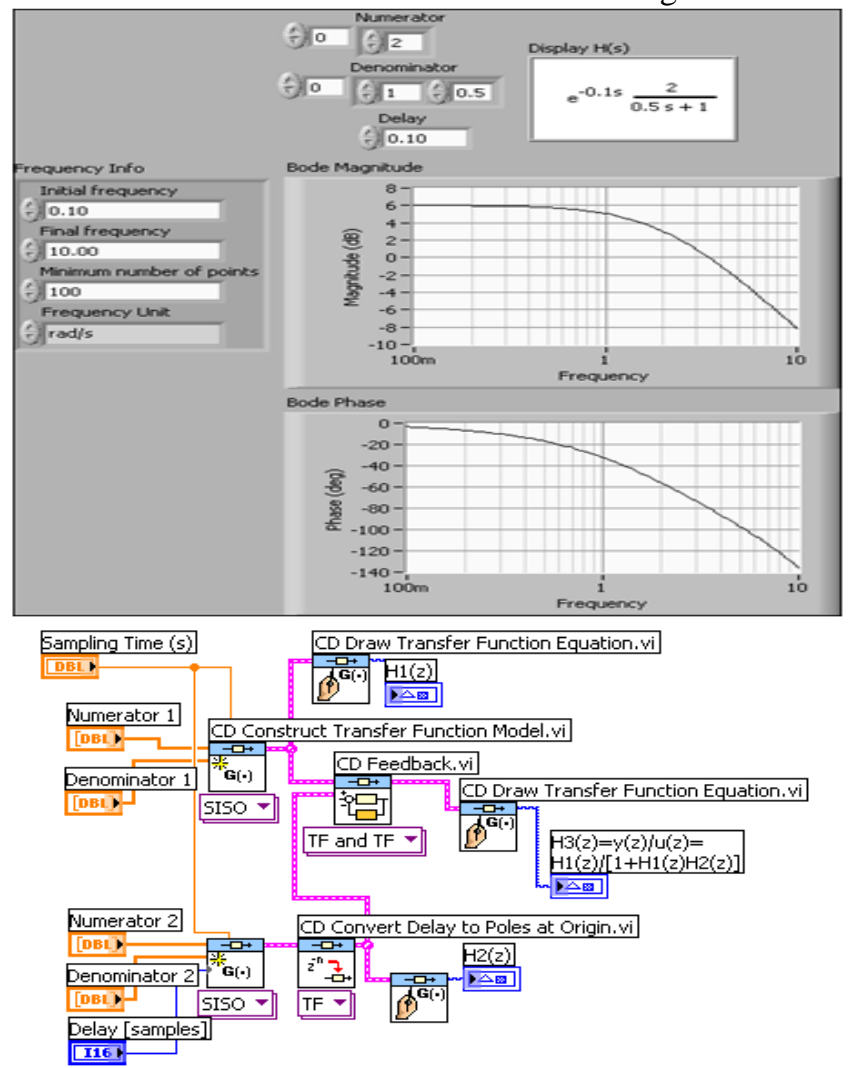

Fig. 3 Front panel and block diagram of frequency_response.vi. 
In the following example, an electric motor (DC) controller is to be modelled, simulated, prototyped and tested with virtual instrumentation and graphical programming language.

Three parameters that affect the motor transfer function are: K (constant), R (resistance), and J (inertia), as shown in Fig.4. It's assumed that the inductance (L) and the friction (b) are very small and negligible. So, the electrical and mechanical diagrams of the plant (DC Motor) are shown in Figure 4.

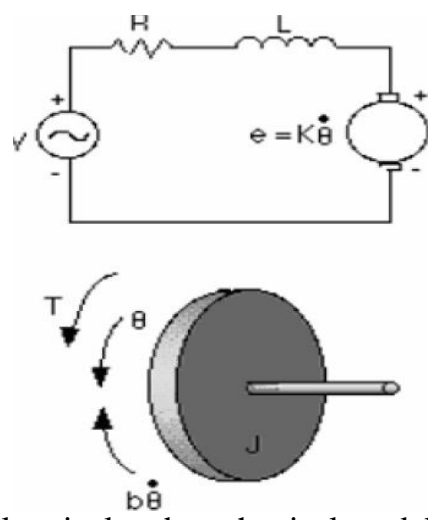

Fig. 4. Electrical and mechanical models of DC motor

Equations for both models (electrical and mechanical) are developed. The circuit diagram of the electric motor consists of a voltage input $(\mathrm{V})$, resistor $(\mathrm{R})$, inductor $(\mathrm{L})$, and back EMF voltage (e) connected in series. In the mechanical diagram, torque $(T)$, position $(\theta)$, friction $(b)$, and inertia. The torque of the motor is proportional to the current consumed by the electric motor. After simplifying and substituting values in the equations for each model, and by taking the Laplace Transform, the resulting transfer function of this plant (motor) is obtained as below

$$
\frac{\text { Angular Speed }}{\text { Input Voltage }}=\frac{\omega(s)}{V(s)}=\frac{K}{J R s+K^{2}}
$$

Now an accurate model of the plant (electric motor) is available, the student proceeds to interactively change the values of $\mathrm{K}, \mathrm{J}$ and $\mathrm{R}$ and evaluate the results in real-time. Once the plant is mathematically modelled, it can then be dynamically simulated and analyzed. For doing this, the student switches to the analysis or Simulation module of the application. At this stage of the design process, the student analyzes the Root-Locus Plot (other options or techniques are available), step response, bode magnitude, and bode frequency of the plant model (Figure 5). In fact, the student can adjust the model parameters and see how the behaviour of the system changes. The root locus method helps evaluate the effect on the roots of the equation by modifying the parameters of the controller. In the example shown bellow, a gain has been put into a feedback loop with the model for the electric motor.

With this first-order system, there is only one pole as per next equation [6].

$s=\frac{-K^{2}}{J R}$

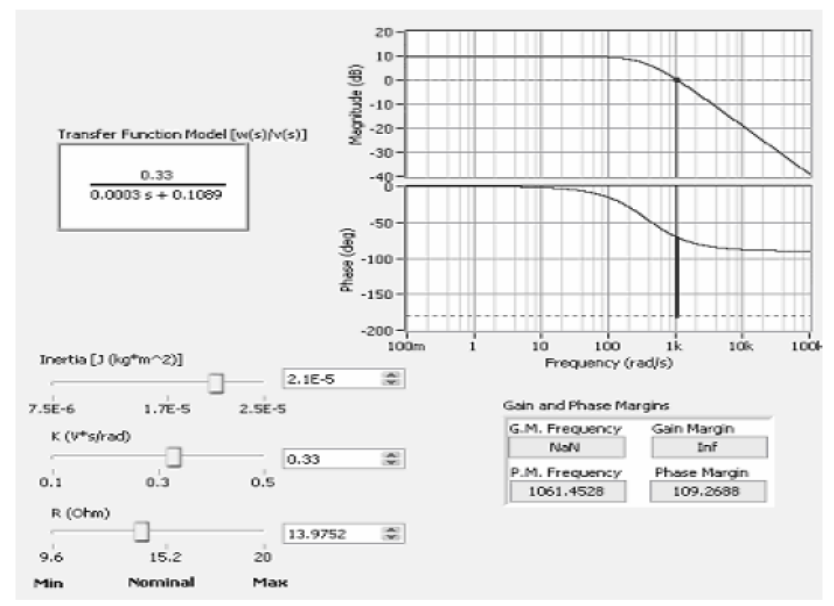

Fig. 5 DC motor model analysis 


\section{Conclusion}

LabVIEW is a powerful environment for developing simulation trainers. Besides the mathematical functions it offers a lot of possibilities for easily creating control panels.

In this paper we presented laboratory practices to be used for students to teach control design subject. We demonstrated use of LabVIEW control design toolkit for teachers and students. It encompasses the different parts of control implementation and instrumentation. Simulated and experimental results can be analysed providing interesting comparisons and the validation of the modelling and controller design.

Using this method, the learning-teaching process becomes an active one. Finally, the fact that students can validate the theory behind control design with a working prototype of the controller-plant system using flexible virtual instruments helps to enhance the education process and help to make engineering education more engaging, enjoyable, fun and effective.

In short, virtual instrumentation improves the quality of education. Students receive while they enjoy to learn, to experiment, and to create.

\section{Journal Papers:}

\section{References}

[1] Murat Tanyel, Dordt College, Enhancing the DSP Toolkit of LabVIEW, Proceedings of the 2002 American Society for Engineering Education Annual Conference \& Exposition.

[2] Nesimi Ertugrul, Towards Virtual Laboratories: a Survey of LabVIEW-based Teaching/Learning Tools and Future Trends.

[3] Vilma A . Oliveira, Manoel L. Aguiar and Wilson da Silva, User - Friendly Computer Software in Control and Instrumentation Teaching and Learning, C.P. 359, 13560-970, São Carlos, SP

[4] Finn Haugen, Introduction to LabVIEW Control Design Toolkit 1.0, January 30, 2005 from Tech Teach.

Website:

[5] What is NI LabVIEW.mht

\section{Journal Papers:}

[6] Igor Alvarado, ISA/IEEE Member, Active Learning of Control Theory Using Virtual Instrumentation, 9th International Conference on Engineering Education $\mathrm{R} 2 \mathrm{H}-1$, July $23-28,2006$. 